\title{
Effect of intraocular scattering in macular pigment optical density measurements
}

\author{
Dimitrios Christaras \\ Alexandros Pennos \\ Harilaos Ginis \\ Pablo Artal
}




\title{
Effect of intraocular scattering in macular pigment optical density measurements
}

\author{
Dimitrios Christaras, ${ }^{\mathrm{a}, \mathrm{b}, *}$ Alexandros Pennos, ${ }^{\mathrm{a}}$ Harilaos Ginis, ${ }^{\mathrm{c}}$ and Pablo Artal ${ }^{\mathrm{a}}$ \\ auniversidad de Murcia, Instituto Universitario de Investigación en Optica y Nanofísica, Laboratorio de Optica, Murcia, Spain \\ bUniversity College London, UCL Institute of Ophthalmology, London, United Kingdom \\ ${ }^{\mathrm{C}}$ Athens Eye Hospital, Department of Research, Athens, Greece
}

\begin{abstract}
Fundus reflectometry is a common in-vivo, noninvasive method to estimate the macular pigment optical density (MPOD). The measured density, however, can be affected by the individual's intraocular scattering. Scattering causes a reduction in the contrast of the fundus image, which in turn leads to an underestimation of the measured density. Intraocular scattering was measured optically in a group of seven young, healthy subjects using the method of optical integration and was subsequently used to correctly estimate the MPOD from fundus images. It was shown that when scattering is not considered, the measured optical density using fundus reflectometry can be underestimated by as high as $16 \%$ for our group of subjects. () The Authors. Published by SPIE under a Creative Commons Attribution 3.0 Unported License. Distribution or reproduction of this work in whole or in part requires full attribution of the original publication, including its DOI. [DOI: 10.1117/1.JBO.23.5.056001]
\end{abstract}

Keywords: intraocular scattering; straylight; macular pigment; fundus imaging

Paper 180050R received Jan. 23, 2018; accepted for publication Apr. 19, 2018; published online May 9, 2018.

\section{Introduction}

Intraocular scattering has a large spatial range, extending well beyond $2 \mathrm{deg}^{1-4}$ and causing an overall contrast decrease in recorded fundus images. This decrease depends on three factors: the wavelength, with the decrease being is stronger at the edges of the visible spectrum, ${ }^{5,6}$ the quality of the ocular optics, and finally the dynamic range of the fundus image.

Scattering in fundus images is manifested in a similar manner as in vision: light originating from the brighter components of the fundus image creates a veil of light over the darker parts of the image. Scattering increases with age in cuartic manner, ${ }^{4}$ but elevated scattering can also be the result of specific ocular diseases, such as corneal edemas and swelling ${ }^{7,8}$ or cataracts affecting the crystalline lens. ${ }^{9,10}$

Macular pigment (MP) is a collective term for the carotenoids lutein, zeaxanthin and mesozeaxanthin, and, as its name suggests, is located mainly, but not exclusively,-at the macula, with its higher concentration observed at the fovea. Its yellow color and the fact that it is located prior to the outer segments of the photoreceptors suggest that MP acts as a short wavelength filter. ${ }^{11}$ Moreover, carotenoids are well known for their antioxidant activity ${ }^{12}$ and, therefore, MP is thought to also act as an antioxidant protective agent in the retina. ${ }^{13-15}$ The lack of it has been correlated to degenerative macular diseases, such as agerelated macular degeneration. ${ }^{16-18}$ In other studies, MP has been related to improved visual function, particularly acuity and visibility. ${ }^{19,20}$

In the recent years, several in-vivo methods for the measurement of the optical density of the MP have been developed, classified in two categories: the optical, or objective, methods and the psychophysical, or subjective, ones. From the optical

*Address all correspondence to Dimitrios Christaras, E-mail: d.christaras@ucl. ac.uk methods, fundus reflectance ${ }^{21,22}$ and fundus autofluorescence ${ }^{23}$ are the most commonly used, with good repeatability and coherent results. Among the psychophysical methods, heterochromatic flicker photometry ${ }^{24,25}$ is the most commonly used, with several commercial devices available. Each of the above methods may suffer from scattering to a different extent, ${ }^{26}$ which depends on the specifics of the setup and the nature of the measurement.

The objective of this work was to evaluate the effect of intraocular scattering in macular pigment optical density (MPOD) measurements using reflectance values from fundus images, recorded using a flood illumination system. In addition, a method for compensation of the effect for more accurate MPOD measurements is proposed.

\section{Methods}

A complete measurement consisted of essentially three distinct steps: (a) the objective reconstruction of the individual's wide angle point spread function (PSF), (b) the acquisition of foveal images at 450 and $550 \mathrm{~nm}$ and their "correction" using the corresponding PSF (calculated previously), and (c) the calculation of the MPOD, through the appropriate formulae. Each step is described in more detail in Secs. 1, 2.2, and 2.3 below.

The instrument used for the reconstruction of the PSF and to capture the fundus images for the measurement of the MPOD is shown in Fig. 1. The setup consisted of an arc lamp backilluminating a mechanical iris (I), which was conjugated to the retina through lenses L1, L2, and L3. For the imaging, light from the fundus was directed to an EMCCD camera with the use of a half mirror, conjugated to the retinal plane through lenses L2, L3, and L4. The wavelength could rapidly switch between 450 and $550 \mathrm{~nm}$ using an interference filter wheel $(\lambda)$ and the appropriate illumination time was achieved with the use of a mechanical shutter $(\mathrm{Sh})$ controlled by a microcontroller. Two appropriately positioned diaphragms (D1, D2) were used to 


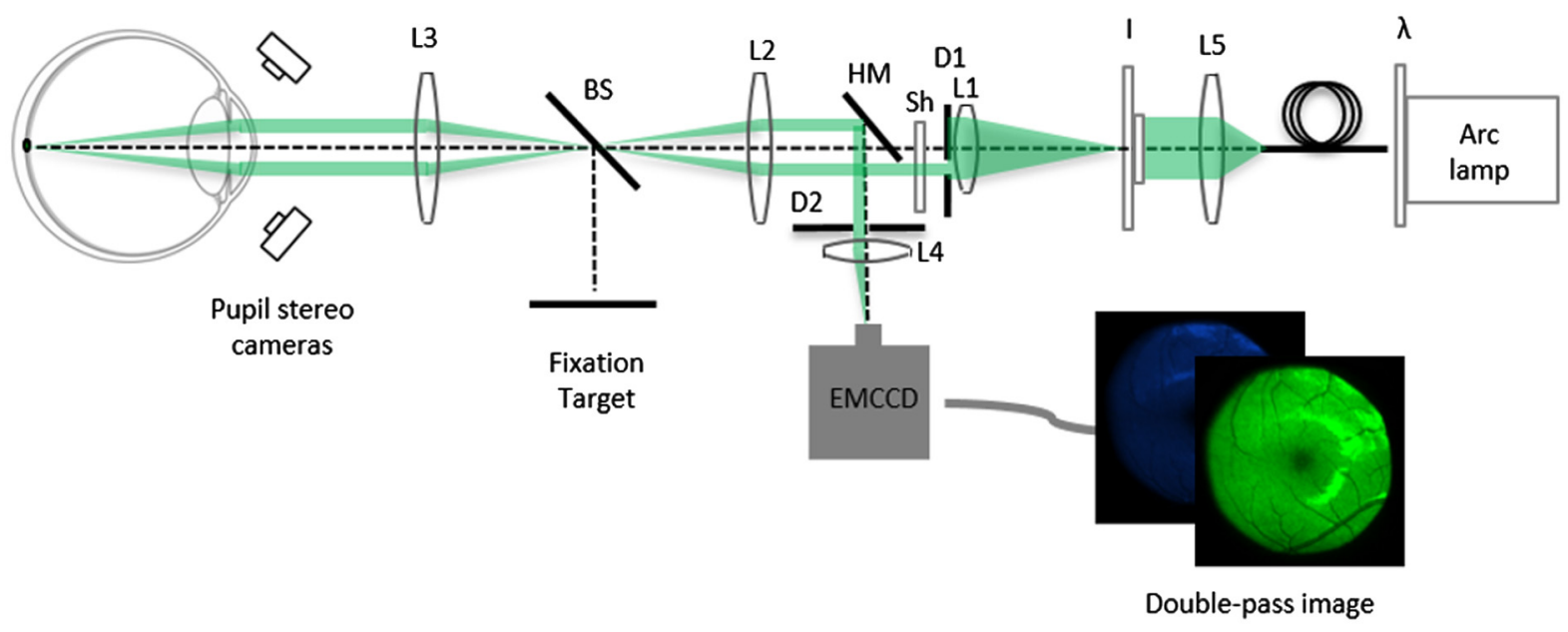

Fig. 1 Optical system for the reconstruction of the wide angle PSF and the recording of the images used in the MPOD measurement.

avoid corneal and lens reflections, ensuring that the captured light originates exclusively from the fundus. The latter is particularly important in scattering measurements. A target located at the retinal plane through beam splitter was used for eye fixation. Finally, a stereo camera system was used for the precise alignment of the pupil. Details on the alignment system can be found in authors' previous works. ${ }^{27,28}$ A more thorough description of the optical system can be found in previous work by Christaras et al. ${ }^{1}$

The high sensitivity of the EMCCD camera allowed us to use low light power for the imaging of the fundus; maximum light power at the corneal plane was measured using a Newport 1815C Power Meter (Newport Corporation, United States) and was found to be $0.135 \mathrm{~mW}$, well below the ISO 15004-2:2007 maximum permissible exposure limits.

\subsection{PSF Calculation}

The scatter estimation was based on the method of optical integration, and it is explained in detail elsewhere. ${ }^{2,5}$ In short, the method consists of the projection of a set of disks of increasing diameter on the retina and the subsequent recording of the double pass image. The double-pass PSF at a specific angle is then calculated by differentiating the intensity value at the center of a recorded disk extending up to that angle. ${ }^{2}$ Therefore, by projecting an adequate number of disks of different size and measuring their reflectance intensity at the center, one can reconstruct the double-pass PSF to the desired spatial extent and resolution. The single-pass PSF can then be calculated by taking the square-root of the Fourier transform of the double-pass PSF. In practice, for a less noisy PSF reconstruction, a monoparametric PSF based on the CIE glare function ${ }^{4}$ was used. A family of PSFs and subsequently of intensity values was simulated for the different parameter values, and the curve that best fitted the intensity data was determined. The corresponding modulation transfer function (MTF) could then be calculated by taking the absolute value of the Fourier transform of the PSF.

Several fundus images were taken prior to the actual measurement, to find the best focus and an appropriate vein-free retinal site for the scattering measurement. Intensity analysis of the images showed no significant reflectance fluctuations, suggesting that photoreceptor reflectance did not change throughout the measurement.

The actual reconstruction of the PSFs for the set of subjects was carried out in a previous study. ${ }^{1}$

\subsection{Acquisition, Registration, and Correction of the Foveal Images}

For the measurement of the MPOD, the procedure consisted of the intensity analysis of two fundus foveal images of $12 \mathrm{deg}$ diameter, taken at $550 \mathrm{~nm}$, where the MP absorption is insignificant, and at $450 \mathrm{~nm}$, where the absorption is close to its maximum. ${ }^{29,30}$ The exposure time was kept below $10 \mathrm{~ms}$ to avoid motion blur and ensure that the recorded intensity range is similar for all subjects and below camera saturation.

In total, five fundus images were acquired at each wavelength and were registered and averaged to increase the signal-to-noise ratio. Subsequently, the images at 550 and $450 \mathrm{~nm}$ were again spatially registered with respect to one another so that each pixel depicted the exact same retinal region. An appropriate filter function was applied in the Fourier domain to all images in order to eliminate irrelevant structures mostly visible in blue ${ }^{31,32}$ and blood vessels absorbing at both blue and green. ${ }^{33}$ The low-pass filter was chosen such that frequencies higher than about 2 cycles/deg would be eliminated, leaving the lower frequencies almost intact. This was done by multiplying the MTF with the function:

$G(f)=e^{-a f^{6}}$

where $a$ is a parameter related to the frequency cut-off limit and $f$ is $f=\sqrt{f_{x}^{2}+f_{y}^{2}}$. The effect of this filter function on a fundus image is shown in Fig. 2.

Subsequently, the images were corrected for intraocular scattering following our compensation method. ${ }^{1}$ Briefly, for the correction of a fundus image, a deconvolution-based technique was used; according to this technique, the appropriate diffractionlimited MTF was divided by the reconstructed "scattering" MTF and the result was multiplied with the image in the Fourier domain. The resulting image was then compensated for the measured scattering effects. 


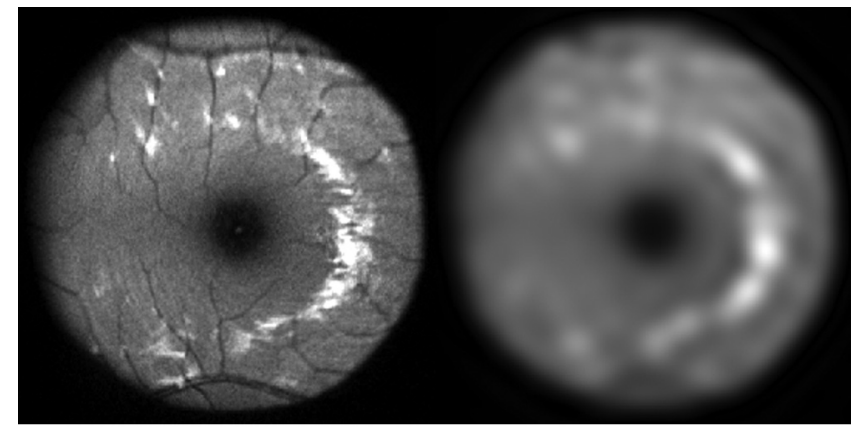

(a)

(b)

Fig. 2 Example of a 12-deg fundus image (a) before and (b) after applying the spatial filtering.

\subsection{MPOD Estimation}

For the estimation of the MPOD, a simplified four-layer fundus model was assumed and the method described by Delori et al. ${ }^{23}$ was followed. In their work, Delori et al. ${ }^{23}$ observed a good correlation between this method and other methods for the measurement of the MP.

The fundus layers, following the direction of light, were: inner limiting membrane (ILM), MP, photoreceptor layer ( $\mathrm{Ph}$ ), retinal pigment epithelium (RPE), Bruch's membrane (BM), and choroid. Assuming that no light is attenuated or reflected before the MP, then the reflectance measured at the camera is related with the MPOD through the relation:

$R(x, y, \lambda)=R^{*}(x, y, \lambda) 10^{-2 D(x, y, \lambda)}$,

where $R^{*}$ is the reflectance of all layers posterior to the MP, $D$ is the MP optical density, and the factor 2 expresses the double pass of the light through the MP. Let $(x, y)$ and $\left(x^{\prime}, y^{\prime}\right)$ be two different retinal sites, then applying Eq. (1) at each site, dividing the two equations and with a little bit of algebra, one obtains

$$
\begin{aligned}
\log \frac{R(x, y, \lambda)}{R\left(x^{\prime}, y^{\prime}, \lambda\right)}= & \log \frac{R^{*}(x, y, \lambda)}{R^{*}\left(x^{\prime}, y^{\prime}, \lambda\right)} \\
& +2\left[D\left(x^{\prime}, y^{\prime}, \lambda\right)-D(x, y, \lambda)\right] .
\end{aligned}
$$

Replacing the optical density difference between the two sites $D_{\mathrm{MP}}(\lambda)=D\left(x^{\prime}, y^{\prime}, \lambda\right)-D(x, y, \lambda)$ and, subsequently, expressing that density difference as a function of the extinction coefficient $D_{\mathrm{MP}}(\lambda)=D_{\mathrm{MP}}(460) K_{\mathrm{MP}}(\lambda)$ from Brown and Wald, ${ }^{29}$ Eq. (2) becomes

$$
\log \frac{R_{\mathrm{P}}(\lambda)}{R_{\mathrm{F}}(\lambda)}=\log \frac{R_{P}^{*}(\lambda)}{R_{F}^{*}(\lambda)}+2 D_{\mathrm{MP}}(460) K_{\mathrm{MP}}(\lambda),
$$

where site $(x, y)$ lies at the perifovea $(\mathrm{P})$ and site $\left(x^{\prime}, y^{\prime}\right)$ at the fovea (F). Applying it for two different wavelengths $\lambda_{1}$ and $\lambda_{2}$ and subtracting one equation from the other, one obtains

$$
\begin{aligned}
\log \frac{R_{\mathrm{P}}\left(\lambda_{1}\right)}{R_{\mathrm{F}}\left(\lambda_{1}\right)} & -\log \frac{R_{\mathrm{P}}\left(\lambda_{2}\right)}{R_{\mathrm{F}}\left(\lambda_{2}\right)}=\log \frac{R_{\mathrm{P}}^{*}\left(\lambda_{1}\right)}{R_{\mathrm{F}}^{*}\left(\lambda_{1}\right)}-\log \frac{R_{\mathrm{P}}^{*}\left(\lambda_{2}\right)}{R_{\mathrm{F}}^{*}\left(\lambda_{2}\right)} \\
& +2 D_{\mathrm{MP}}(460)\left[K_{\mathrm{MP}}\left(\lambda_{1}\right)-K_{\mathrm{MP}}\left(\lambda_{2}\right)\right] .
\end{aligned}
$$

Assuming now that the reflection spectrum at the perifovea is proportional to the reflection spectrum at the fovea, then that the ratio $\frac{R_{\mathrm{P}}^{*}(\lambda)}{R_{\mathrm{F}}^{*}(\lambda)}$ is constant for all wavelengths, and therefore, Eq. (4) is simplified to

$$
\begin{aligned}
\log \frac{R_{\mathrm{P}}\left(\lambda_{1}\right)}{R_{\mathrm{F}}\left(\lambda_{1}\right)} & -\log \frac{R_{\mathrm{P}}\left(\lambda_{2}\right)}{R_{\mathrm{F}}\left(\lambda_{2}\right)}=2 D_{\mathrm{MP}}(460)\left[K_{\mathrm{MP}}\left(\lambda_{1}\right)\right. \\
& \left.-K_{\mathrm{MP}}\left(\lambda_{2}\right)\right] .
\end{aligned}
$$

Finally, solving Eq. (5) for $D_{\mathrm{MP}}(460)$, one obtains

$$
D_{\mathrm{MP}}(460)=\frac{0.5}{K_{\mathrm{MP}}\left(\lambda_{1}\right)-K_{\mathrm{MP}}\left(\lambda_{2}\right)}\left\{\log \frac{R_{\mathrm{P}}\left(\lambda_{1}\right)}{R_{\mathrm{P}}\left(\lambda_{2}\right)}+\log \frac{R_{\mathrm{F}}\left(\lambda_{2}\right)}{R_{\mathrm{F}}\left(\lambda_{1}\right)}\right\},
$$

where in our experiment $\lambda_{1}=550 \mathrm{~nm}$ and $\lambda_{2}=450 \mathrm{~nm}$.

The values for the extinction coefficients were extracted from Brown's data ${ }^{29}$ after integrating over the spectrum of each interference filter and they were calculated to be $K_{\mathrm{MP}}(450)=$ 0.9038 and $K_{\mathrm{MP}}(550)=0.0089$.

There are a number of different metrics used for the measurement of the MPOD like the median, the peak value, the weighted average, etc. However, no golden standard exists and different devices use different metrics. We used the average value for an area of $1 \mathrm{deg}$ around the center of the fovea as the value for the MPOD. This is similar to the field size used in some commercial psychophysical devices. ${ }^{34}$

We performed the series of measurements in seven healthy volunteers, two females and five males, all Caucasians, with normal vision and no known ocular diseases. The younger subject was 23 years old and the older 34 years old and the average age was 29 years old. Four subjects had light iris pigmentation (blue or green) and three subjects had heavy iris pigmentation (brown). All the research and measurements followed the tenets of the Declaration of Helsinki and were approved by the local ethics committee. Informed consent was obtained from the subjects after they were fully informed about the nature of the measurements.

In order to see the effect of scattering in the MPOD measurement, the scattering compensation technique ${ }^{1}$ was applied for both the original images without any enhancement and the images after the scattering compensation. The resulted MPOD was then compared, and the relative effect of the correction was estimated.

\section{Results}

For each subject, two PSFs, one at each wavelength, were calculated in our previous work. ${ }^{1}$ The computed scatter parameter is shown in Table 1. The reconstructed PSFs at 450 and $550 \mathrm{~nm}$ for two subjects are shown in Fig. 3.

The profile of the MPOD spatial distributions for all seven subjects is presented graphically in Fig. 4 for both cases, i.e., with scattering compensation (red line) and without scattering compensation (blue line). Table 1 shows the values for the average MPOD for $1 \mathrm{deg}$ before (fifth column) and after (sixth column) scattering compensation, as well as the relative change (last column). As seen in the table, the underestimation of the MP when scattering is not considered ranges between $5.2 \%$ and $16 \%$, with an average of $9.4 \%$. The average MPOD before the compensation was found to be $0.176 \pm 0.058$ and $0.192 \pm$ 0.064 after compensation. The importance of the effect depends on the amount of intraocular scattering at blue and green, their 
Table 1 MPOD values before and after scattering compensation as well as the relative change between the two measurements for seven subjects.

\begin{tabular}{lcccccc} 
Subject & Age & $\begin{array}{c}\text { Scattering parameters } \\
\text { (blue) }\end{array}$ & $\begin{array}{c}\text { Scattering parameters } \\
\text { (green) }\end{array}$ & MPOD (no comp.; D.U.) & MPOD (comp.; D.U.) & Relative increase (\%) \\
\hline S1 & 31 & 1.21 & 0.98 & 0.151 & 0.160 & 6.0 \\
S2 & 31 & 1.35 & 0.96 & 0.219 & 0.254 & 16.0 \\
S3 & 34 & 1.29 & 1.01 & 0.123 & 0.137 & 11.4 \\
S4 & 23 & 1.32 & 1.32 & 0.200 & 0.216 & 8.0 \\
S5 & 29 & 1.15 & 1.05 & 0.211 & 0.222 & 5.2 \\
S6 & 25 & 1.20 & 0.96 & 0.243 & 0.263 & 8.2 \\
S7 & 30 & 1.31 & 1.15 & 0.082 & 0.091 & 11.0 \\
\hline
\end{tabular}
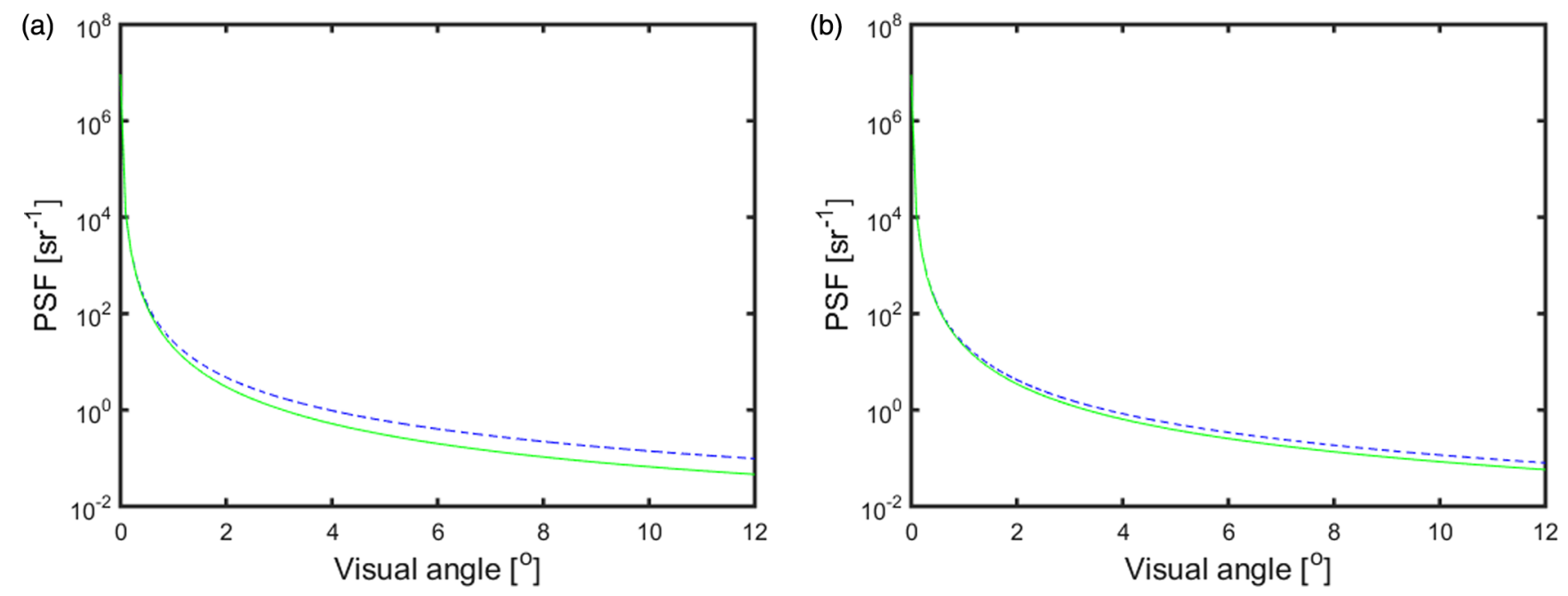

Fig. 3 (a) and (b) Reconstructed PSFs at $450 \mathrm{~nm}$ blue/dashed and $550 \mathrm{~nm}$ (green) for two different subjects.

difference, specular reflections in the image, as well as the spatial characteristics of the MP itself.

\section{Discussion}

In this work, the effect of intraocular scattering on MPOD measurements was quantified and, subsequently, compensated using an image processing technique. When the corrected images were used for the measurement of the MPOD, a mean increase of $9.4 \%$ in the value was observed for a set of seven young and healthy subjects. Regardless of the possible errors in the reflectometric estimation of MPOD, the incorporation of straylight in the calculation will result in an increase of the estimated value by a factor on the order of $16 \%$. This increase may be higher in eyes with elevated straylight such as in eyes with cataract.

Several assumptions were made for the MPOD extraction from the fundus images. The most important was treating the fundus posterior to the MP as a uniform reflecting surface. For short and middle wavelengths below $580 \mathrm{~nm}$, reflections occur mostly at the RPE and at $\mathrm{BM}^{35,36}$ and, therefore, melanin density at the RPE affects the amount of reflected light. RPE melanin is not constant throughout the fundus but exhibits a higher density at the fovea and, therefore, it has some effect in MPOD from reflectometry measurements. Nevertheless, Delori et al. ${ }^{23}$ found a contribution to the MPOD measurement of 0.03 D.U., concluding thus that the melanin difference across the fundus has a limited effect on the estimation of the MPOD using the reflectometry method. Reflections occurring prior to the $\mathrm{RPE}$ at the $\mathrm{Ph}$, on the other hand, would not be affected by melanin density changes. Another possible scenario is that part of reflected light, however, originates from scattering by the Henle fibers, i.e., where the MP is believed to lie, ${ }^{37}$ and hence leading to a reduced double-pass through the MP and consequently an additional cause to further underestimate the MPOD.

On the reconstruction of the PSF, it is important to note that in our PSF manipulations throughout the method of optical integration, the phase transfer function is assumed to be 0 . This is attributed to the fact that the PSF resulting from scattering is considered as rotationally symmetric, and therefore its Fourier transform will be a real function.

Regarding the directionality of the photoreceptors, because of the pupil configuration used in the system, illuminating and imaging angles are relatively large (about $5 \mathrm{deg}$ ) and wave-guiding is relatively low at those angles and, therefore, the SC effect is negligible. ${ }^{38}$ Tests prior to the experiment supported this fact.

There is yet another important factor that could lead to a wrong estimation of the MPOD using the reflectometry method: 
(a)
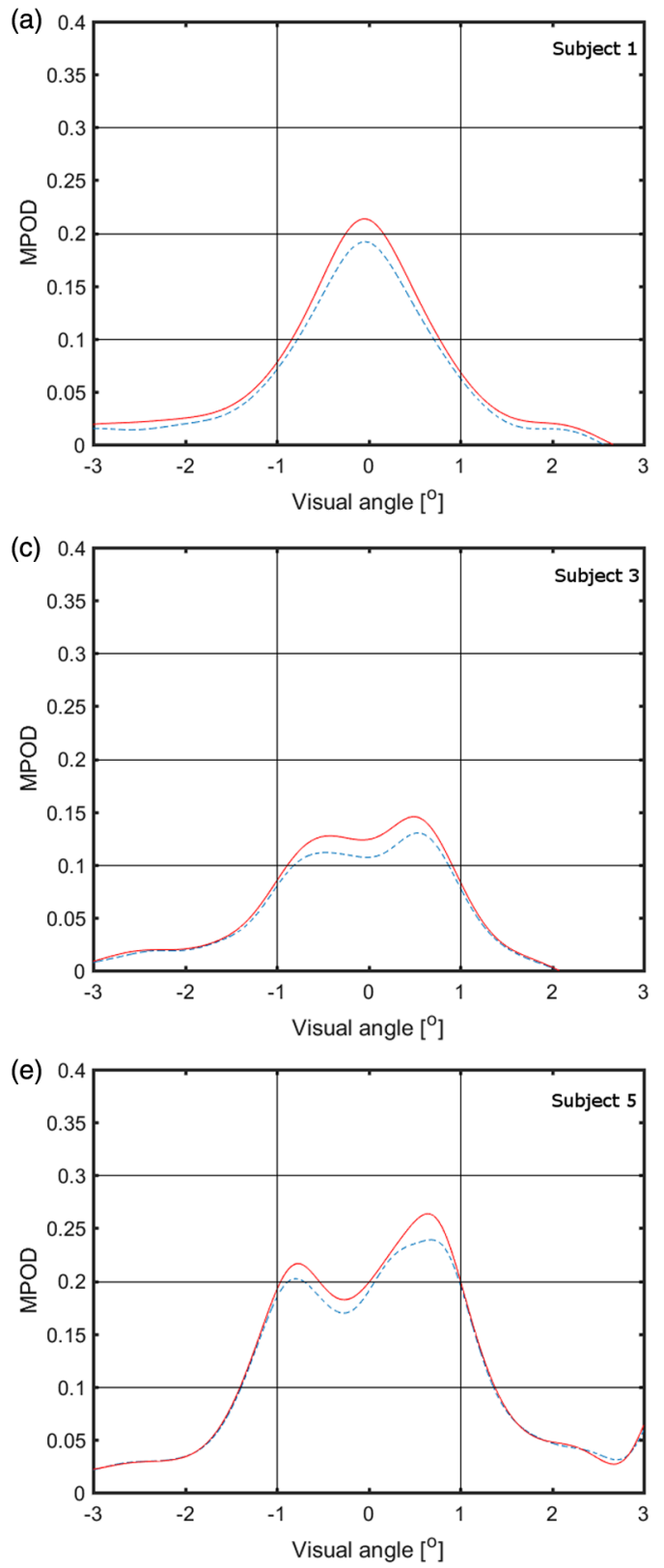

(b)

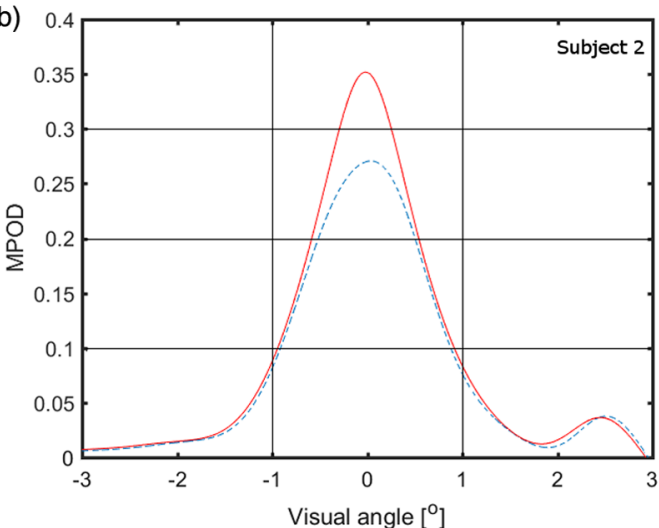

(d)

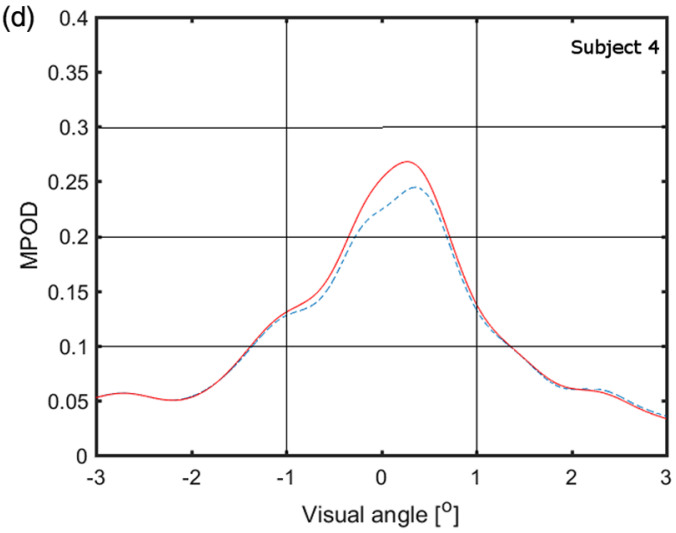

(f)

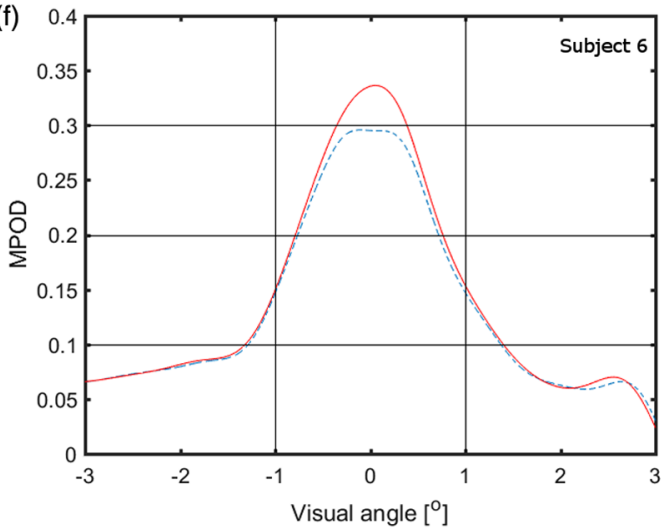

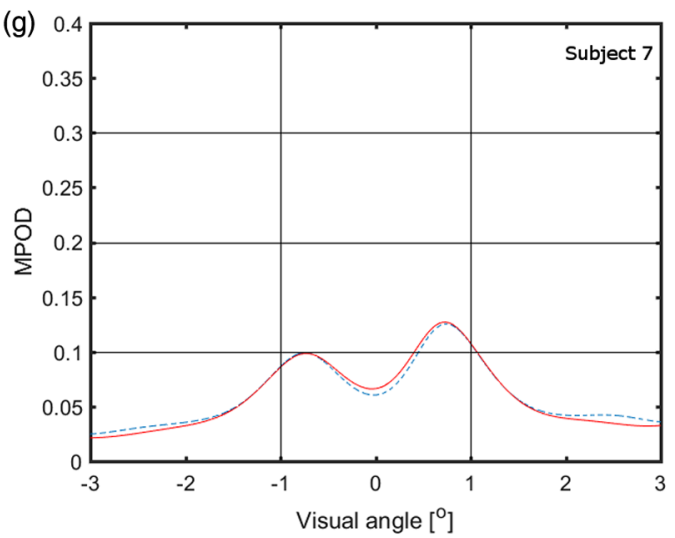

Fig. 4 MPOD nasal-temporal profile before blue/dashed and after (red) scattering compensation for the seven subjects. All profiles were centered at the fovea. 
specular reflections, mainly taking place at the interface between the aqueous humor and the ILM. ${ }^{35}$ Those specular reflections depend on the parameters of the system, such as the incident angle of the rays and the curvature of the fundus. Moreover, they are known to be consistently higher in younger subjects. $^{23,39}$ They are observed in several foveal images in flood illumination systems with unpolarized light, especially at middle and short wavelengths at about $3 \mathrm{deg}$ at the perifovea (see Fig. 2). Those reflections may affect the measurement to some extent, since they act as a glare source, significantly increasing the dynamic range of the image. This was dealt with by ensuring that the image pixels at the sites where the specular reflections occur, were not saturated, though they were kept close to saturation in order to achieve the best possible dynamic range for the rest of the image. The use of cross polarizers could efficiently deal with specular reflections, but it was avoided because it would significantly reduce the available light at the camera and it could potentially have an effect on the measurement of the MP since MP is known to have a polarization preference. $^{40}$

Finally, it needs to be noted that not all MPOD measurement techniques suffer at the same degree from scattering. In this study, only the effect of scattering for a flood illumination system is examined. For a rough estimate on how scattering affects different methods see Table 2 in the work of Leung. ${ }^{26}$ As a rule of thumb, one can expect the higher impact to be observed in flood illumination systems, a medium to low impact in psychophysical methods, and no significant impact in systems with confocality.

\section{Conclusions}

Intraocular scattering was found to affect the estimations of MP optical density when using fundus reflectometry. We showed that, even for a group of young subjects with low amounts of straylight, the effect of scattering in the measurement of the MPOD using this method could be up to $16 \%$. Our method can compensate for this effect and provide scatter-free values for the MPOD.

\section{Disclosures}

The authors declare that there are no conflicts of interest related to this article.

\section{Acknowledgments}

This research has been supported by the European Research Council Advanced Grant ERC-2013-AdG-339228 (SEECAT), Secretaría de Estado e Investigación, Desarrollo e Innovación (SEIDI) FIS2016-76163-R; Fundación Séneca-Agencia de Ciencia y Tecnología de la Región de Murcia (grant 19897/GERM/15), and the European Regional Development Fund (EU-FEDER).

\section{References}

1. D. Christaras et al., "Intraocular scattering compensation in retinal imaging," Biomed. Opt. Express 7(10), 3996-4006 (2016).

2. H. Ginis et al., "The wide-angle point spread function of the human eye reconstructed by a new optical method," J. Vis. 12(3), 20 (2012).

3. D. Christaras et al., "Scattering contribution to the double-pass PSF using Monte Carlo simulations," Ophthalmic Physiol. Opt. 37(3), 342-346 (2017).

4. J. J. Vos and T. J. T. P. Van Den Berg, "The large angle course of the disability glare function and its attribution to components of ocular scatter," CIE Collect. Color Vision 124(2), 11-29 (1997).
5. H. Ginis et al., "Wavelength dependence of the ocular straylight," Invest. Ophthalmol. Visual Sci. 54(5), 3702-3708 (2013).

6. J. E. Coppens, L. Franssen, and T. J. T. P. Van Den Berg, "Wavelength dependence of intraocular straylight," Exp. Eye Res. 82(4), 688-692 (2006).

7. D. B. Elliott et al., "Relative sensitivity of clinical tests to hydrophilic lens-induced corneal thickness changes," Optom. Vision Sci. 70(12), 1044-1048 (1993).

8. K. M. Meek et al., "Transparency, swelling and scarring in the corneal stroma," Eye (Lond.) 17(8), 927-936 (2003).

9. J. J. Vos, "Disability glare - a state of the art report," CIE 3(2), 39-53 (1984).

10. D. B. Elliott and M. A. Bullimore, "Assessing the reliability, discriminative ability, and validity of disability glare tests," Invest. Ophthalmol. Visual Sci. 34(1), 108-119 (1993).

11. D. M. Snodderly, J. D. Auran, and F. C. Delori, "The macular pigment II. Spatial distribution in primate retinas," Invest. Ophthalmol. Visual Sci. 25(6), 674-685 (1984).

12. W. Stahl and H. Sies, "Antioxidant activity of carotenoids," Mol. Aspects Med. 24(6), 345-351 (2003).

13. E. Loane et al., "The rationale and evidence base for a protective role of macular pigment in age-related maculopathy," Br. J. Ophthalmol. 92, 1163-1168 (2008).

14. F. Khachik, P. S. Bernstein, and D. L. Garland, "Identification of lutein and zeaxanthin oxidation products in human and monkey retinas," Invest. Ophthalmol. Visual Sci. 38(9), 1802-1811 (1997).

15. T. Berendschot, "Fundus reflectance-historical and present ideas," Prog. Retinal Eye Res. 22(2), 171-200 (2003).

16. S. Beatty et al., "Macular pigment and age related macular degeneration," Br. J. Ophthalmol. 83(83), 867-877 (1999).

17. S. Beatty et al., "Macular pigment and risk for age-related macular degeneration in subjects from a Northern European population," Invest. Ophthalmol. Visual Sci. 42(2), 439-446 (2001).

18. J. S. L. Tan et al., "Dietary antioxidants and the long-term incidence of age-related macular degeneration. The blue mountains eye study," Ophthalmology 115(2), 334-341 (2008).

19. B. R. Wooten and B. R. Hammond, "Macular pigment: influences on visual acuity and visibility," Prog. Retinal Eye Res. 21(2), 225-240 (2002).

20. J. Loughman et al., "Macular pigment and its contribution to visual performance and experience," J. Optom. 3(2), 74-90 (2010).

21. T. T. J. M. Berendschot, "Imaging the macular pigment," in Medical Retina, F. G. Holz and R. F. Spaide, Eds., pp. 51-68, SpringerVerlag, Berlin, Heidelberg, Germany (2010).

22. T. T. J. M. Berendschot and D. Van Norren, "Objective determination of the macular pigment optical density using fundus reflectance spectroscopy," Arch. Biochem. Biophys. 430, 149-155 (2004).

23. F. C. Delori et al., "Macular pigment density measured by autofluorescence spectrometry : comparison with reflectometry and heterochromatic flicker photometry," J. Opt. Soc. Am. A 18(6), 1212-1230 (2001).

24. B. R. Wooten et al., "A practical method for measuring macular pigment optical density," Invest. Ophthalmol. Visual Sci. 40(11), 2481-2489(1999).

25. D. M. Snodderly, "Macular pigment measurement by heterochromatic flicker photometry in older subjects: the carotenoids and age-related eye disease study," Invest. Ophthalmol. Visual Sci. 45(2), 531-538 (2004).

26. I. F. Leung, "Macular pigment: new clinical methods of detection and the role of carotenoids in age-related macular degeneration," Optometry 79, 266-272 (2008).

27. H. Ginis et al., "Compact optical integration instrument to measure intraocular straylight," Biomed. Opt. Express 5(9), 3036 (2014).

28. D. Christaras, Light Scattering Phenomena in Vision and in Imaging, Universidad de Murcia, Murcia, Spain (2016).

29. P. K. Brown and G. Wald, "Visual pigments in human and monkey retinas," Nature 200(4901), 37-43 (1963).

30. D. M. Snodderly et al., "The macular pigment. I. Absorbance spectra, localization, and discrimination from other yellow pigments in primate retinas," Invest. Ophthalmol. Visual Sci. 25(6), 660-673 (1984).

31. R. W. Knighton, S. G. Jacobson, and C. M. Kemp, "The spectral reflectance of the nerve fiber layer of the macaque retina," Invest. Ophthalmol. Visual Sci. 30(11), 2393-2402 (1989).

32. R. W. Knighton and X. R. Huang, "Directional and spectral reflectance of the rat retinal nerve fiber layer," Invest. Ophthalmol. Visual Sci. 40(3), 639-647 (1999). 
33. E. J. van Kampen and W. G. Zijstra, "Spectrophotometry of hemoglobin and hemoglobin derivatives," Adv. Clin. Chem. 23, 199-257 (1983).

34. J. Loughman et al., "An evaluation of a novel instrument for measuring macular pigment optical density: the MPS 9000," Acta Ophthalmol. 90(2), e90-e97 (2012).

35. F. C. Delori and K. P. Pflibsen, "Spectral reflectance of the human ocular fundus," Appl. Opt. 28(6), 1061-1077 (1989).

36. J. M. Gorrand, R. Alfieri, and J. Y. Boire, "Diffusion of the retinal layers of the living human eye," Vision Res. 24(9), 1097-1106 (1984).

37. R. A. Bone and J. T. Landrum, "Macular pigment in henle fiber membranes: a model for Haidinger's brushes," Vision Res. 24(2), 103-108 (1984).
38. P. J. Delint, T. T. Berendschot, and D. van Norren, "Local photoreceptor alignment measured with a scanning laser ophthalmoscope," Vision Res. 37(2), 243-248 (1997).

39. J. M. Gorrand and F. C. Delori, "Reflectance and curvature of the inner limiting membrane at the foveola," J. Opt. Soc. Am. A 16(6), 1229-1237 (1999).

40. R. A. Bone, "The role of the macular pigment in the detection of polarized light," Vision Res. 20(3), 213-220 (1980).

Biographies for the authors are not available. 